\section{Lehrbuch der Palliativmedizin}

\section{Eberhard Aulbert, Friedemann Nauck u. Lukas Radbruch}

(Hrsg.)

Schattauer Verlag, Stuttgart, 2007, 2., überarb. u. erw. Aufl., 1423 S., 225 Abb., 208 Tab., 129,00 EUR

ISBN 978-3-7945-2361-0

Mit Recht gilt dieses Buch seit seinem ersten Erscheinen 1997 als Standardwerk der Palliativmedizin für Ärzte, Pflegende und psychosoziale Berufsgruppen, die sich der Betreuung Schwerstkranker und Sterbender sowie deren Angehöriger widmen. Es ist das Oxford Textbook of Palliative Medicine für den deutschsprachigen Raum. 10 Jahre nach der Erstausgabe ist das Lehrbuch der Palliativmedizin nunmehr in der zweiten Auflage erschienen.

Die zweite Auflage wurde unter Berücksichtigung der Entwicklungen in der Palliativmedizin aktualisiert, inhaltlich neu und straffer gegliedert und thematisch erweitert. So wurde der terminalen, palliativen Sedierung, dem Fatigue-Syndrom, der Forschung in der Palliativmedizin, der Patientenautonomie und der Evaluation von Lebensqualität jeweils ein neues Kapitel gewidmet. Der zunehmenden Bedeutung der Palliativmedizin bei nicht-onkologischen internistischen Erkrankungen, in der Geriatrie und in der Pädiatrie wurde umfassender Rechnung getragen. Das Kapitel über gastrointestinale Symptome wurde durch einen bereichernden Anhang über Kernaussagen für die palliativmedizinische Behandlung erweitert. Bedauerlich ist, dass bei neuer Autorenschaft dieses Kapitels Text und Abbildungen weitestgehend aus der Erstauflage übernommen wurden, ohne Nennung der Erstautoren.

Insgesamt gesehen jedoch sollte diese Neuauflage des Lehrbuchs der Palliativmedizin einen festen Platz in der Fachbücherei eines jeden palliativmedizinisch oder palliativpflegerisch Tätigen haben.

Reiner Hartenstein, München

\section{Fragen und Antworten zum Mammakarzinom Ein Ratgeber der Projektgruppe Mammakarzinom, Tumorzentrum München}

Hansjörg Sauer $u$. Wolfgang Janni (Hrsg.)

Zuckschwerdt Verlag, München, 2007, 177 S., 14,90 EUR

ISBN 978-3-88603-924-1

Das Buch «Fragen und Antworten zum Mammakarzinom» ist der erste Patient(inn)en-Ratgeber der Projektgruppe Mammakarzinom des Tumorzentrums München zum Thema Brustkrebs. Es basiert auf zahlreichen Fragen, die Patientinnen in den letzten Jahren über ein Frageportal der Projektgruppe Mammakarzinom gestellt haben. Eine repräsentative Auswahl dieser Fragen zu den verschiedensten Aspekten der Erkrankung von z.B. «Risikofaktoren» und «Früherkennung» über «Operation», «medikamentöse Therapie», «Strahlentherapie» sowie «supportive Maßnahmen» bis hin zu spezielleren Fragestellungen wie «Schwangerschaft und Mammakarzinom», «Männer mit Mammakarzinom» und «alternative Behandlungsangebote» wird jeweils von ausgewiesenen Experten beantwortet. Erklärtes Ziel dieses Ratgebers ist, Patientinnen, die mit der Diagnose Brustkrebs konfrontiert werden, in dieser schwierigen Lebenssituation durch fundierte und ausgewogene Informationen $\mathrm{zu}$ unterstützen und so ein Verständnis der Erkrankung und ihrer Behandlung zu ermöglichen.

Der Ratgeber ist im Frage-Antwort-Stil strukturiert. Dabei beginnen die einzelnen Kapitel mit Fragen zu allgemeinen Aspekten der Brustkrebs-Erkrankung, so dass zunächst sehr grundlegende Zusammenhänge dargestellt werden. Zu jedem Thema werden jedoch auch speziellere Fragen formuliert, zum Teil in Form konkreter (und meist übertragbarer) Fallbeispiele. So werden die häufigsten Fragen, die die Betroffenen intensiv beschäftigen und mit denen man als behandelnder Arzt jeden Tag in der Brustsprechstunde konfrontiert wird, systematisch behandelt. Besonders wertvoll ist, dass zahlreiche Themen, die den Alltag und Lebenswandel der Patientinnen betreffen, wie zum Beispiel «Krebsdiäten» oder klimakterische Beschwerden nach Mammakarzinom-Therapie, beleuchtet werden. Auch komplementärmedizinische Maßnahmen bzw. alternative Therapien (Misteltherapie, Vitamine, Spurenelemente, japanische Pilze, Sojaprodukte etc.), in die viele Patientinnen erfahrungsgemäß große Hoffnungen setzen, werden sachlich-kritisch diskutiert und der oft fragliche Nutzen bzw. die Bedeutung prospektiv-randomisierter Behandlungsstudien zur Wirksamkeitsbewertung dargestellt. Ausgesprochen hilfreich sind ferner die praktischen Tipps beispielsweise zur aktuellen Heilmittelverordnung, in denen neben den Verordnungsvorschriften auch konkrete Hinweise, wie bestimmte Heilmittel rezeptiert werden müssen, damit eine Kostenerstattung seitens der Krankenkasse stattfindet, gegeben werden. Im Anhang des Ratgebers finden sich eine umfassende Liste von wichtigen Medikamenten in der Tumortherapie, eine Adressensammlung von Stellen, an die Betroffene sich für Unterstützung und Information wenden können und ein Glossar mit Erläuterungen der wichtigsten Fachbegriffe. Das Stichwortverzeichnis, welches sowohl Fachtermini als auch für den Laien gut verständliche Ausdrücke ( «Sentinel Lymph Node»/«Wächterlymphknoten»; «Obstipation»/«Verstopfung») enthält und so ein schnelles Nachschlagen von Informationen ermöglicht, rundet das gelungene Bild des Buches ab.

Der Patient(inn)enratgeber verschafft einen gut verständlichen Überblick über die Diagnose Brustkrebs und die verschiedenen Behandlungsmöglichkeiten. Er hält, was er verspricht, und ist sicher vielen neu an Brustkrebs erkrankten Frauen, die sich umfassend und vor allem medizinisch fundiert informieren wollen, eine große Hilfe.

\section{KARGER}

Fax +497614520714

E-mail Information@Karger.de www.karger.com/onk

www.karger.com

www.karger.com/onk

\section{(c) 2008 S. Karger GmbH, Freiburg}

Accessible online at: 